\title{
Assessment of relationship between the use of household products and atopic dermatitis in Seoul: focused on products with associated risks
}

\author{
Jihee Choi' ${ }^{1}$, Jeonghoon Kim ${ }^{1}$, KyooSang Kim ${ }^{1,2}$ [C] \\ 'Department of Environmental Health Research, Seoul Medical Center, Seoul, Republic of Korea; ${ }^{2}$ Department of Occupational and Environmental \\ Medicine, Seoul Medical Center, Seoul, Republic of Korea
}

\begin{abstract}
The purpose of this study was to evaluate the relationship between the use of certain household products (HPs) at home and atopic dermatitis (AD) in adults. The study was conducted on 1,500 households in Seoul, South Korea. We obtained information on general characteristics, the use of HPs, and AD through an online panel survey in July 2018. HPs were selected as 23 kinds of products with associated risks that are management targets of the Ministry of Environment. The international study of asthma and allergies in childhood was used for the $A D$ questionnaires. Logistic regression analysis was used to identify AD affected by the use of HPs. Average number of used HPs was 13.44 out of 23, and average usage frequency of HPs was 3.52 times a month at home in Seoul for the last 1 year. Compared with subjects with a low number of HPs used (reference), subjects with a high number of used HPs (4th quartile) were more likely to have lifetime diagnosis of $A D$ (odds ratio $(O R)=1.77,95 \%$ confidence interval $(\mathrm{Cl}) ; 1.23-2.54$ ), symptoms of $A D$ in the last 12 months $(O R=2.66,95 \% C l ; 1.92-3.70)$, and treatment of $A D$ in the last 12 months $(O R=2.37,95 \%$ $\mathrm{Cl} ; 1$ 1.48-3.80). Compared with subjects with a low HPs usage frequency (reference), subjects with a high HPs usage frequency (4th quartile) were more likely to have lifetime diagnosis of $A D(O R=1.88,95 \% \mathrm{Cl} ; 1.31-2.70)$, symptoms of $A D$ in the last 12 months $(O R=2.14,95 \% \mathrm{Cl} ; 1.54-2.96)$, and treatment of $A D$ in the last 12 months $(O R=2.23,95 \% \mathrm{Cl} ; 1.39-3.60)$. Therefore, the use of HPs was significantly associated with AD. The findings of this study might be useful as basic data for managing allergic diseases and establishing preventive measures.
\end{abstract}

Keywords: Adult, Atopic dermatitis, Household product, Seoul

\section{INTRODUCTION}

Household products (HPs) are constantly being used in daily living, to the extent that a new phrase "homo chemicus" has been coined, meaning humans living in a state of dependence on chemical products [1]. Since the humidifier disinfectant accident in 2011, awareness of the risk of HPs has grown, as incidents involving chemicals in HPs continue to occur, such as the inclusion of humidifier disinfectants in some toothpastes, the presence of pesticide in eggs, the discovery of hazardous substances in sanitary napkins, methanol from vehicles in washers, and radon in beds [2]. In fact, according to the

Received: April 8, 2019 Accepted: June 21, 2019

Corresponding author: KyooSang Kim

156 Sinnae-ro, Jungnang-gu, Seoul 02053, Republic of Korea

E-mail: kyoosang@daum.net

Funding: This study was supported by Seoul Medical Center Research Institute, Republic of Korea

This article is available from: http://e-eht.org/
Korea Consumer Agency (KCA), 1,529 cases of the risks of HPs were received through consumer surveillance system in the 3 -year period from 2014 to 2016 , with $46.5 \%$ more cases in 2016 than in 2014 [3].

HPs are continuously produced according to the purpose and use of each manufacturing company, and there are many chemicals in these HPs that are not properly managed [4]. According to the National Institute of Environmental Research (NIER), 682 products, which account for about $50 \%$ of the 1,369 HPs in total, were reported as using nanomaterials [5]. In the study of ingredients and hazardous substances contained in detergents and disinfectants, 38 of the 163 ingredients were classified as dangerous substances, and some of them have been shown to be harmful or carcinogenic in oral, transdermal, and inhalation exposure [6]. In an overseas study, 133 kinds of volatile organic compounds were detected among 25 HPs such as laundry products, personal products, cleaning products, and fragrances [7]. In addition, since there are still 
cases in which the labeling of product ingredients is insufficient, consumers may not be properly informed of the harmful chemical substances contained in HPs, as was the case in the humidifier disinfectant incident, and may be exposed to unexpected health effects [8].

A previous study has reported that chemicals in HPs can adversely affect health, resulting in symptoms such as skin rash, allergies, eye irritation, and dyspnea [9]. Of these, atopic dermatitis $(\mathrm{AD})$ with severe itching and dry symptoms is one of the most common skin diseases not only for infants and children but also for adults in Korea [10], and the prevalence of $\mathrm{AD}$ continues to grow with the rapid increase of allergic diseases worldwide [11]. According to Statistics Korea (KOSTAT), rates of $\mathrm{AD}$ diagnosis in adults aged 19 and over increased from $2.4 \%$ in 2007 to $4.1 \%$ in 2017 [12]. The cause of $\mathrm{AD}$ has not yet been clearly identified, but it is known that various environmental or genetic factors interact with each other and contribute in a complex manner [13]. Significantly, it has been reported that environmental factors are related to the increase of exposure to antigens due to Korea's westernization and changes in the living environment, as well as the presence of allergens such as automobile exhaust, dust, particle matter, etc. [14].

While studies on adult $\mathrm{AD}$ continue to be conducted abroad [15], there has been a lack of interest in adult AD in Korea compared to children $\mathrm{AD}$. As such, the purpose of this study is to identify usage status of HPs at home in Seoul, targeting products with associated risks that are management targets of the Korean Ministry of Environment (ME), and to evaluate the relationship between the use of HPs and AD in adults.

\section{MATERIALS AND METHODS}

\section{Study Population.}

An online survey was conducted on 1,500 households who lived in Seoul for more than 1 year, using online participants who voluntarily registered with a survey company for 20 days from July 11 to July 30, 2018. To ensure that the survey sample was representative of households in Seoul, we used the statistical data of the Population and Housing Census in 2015, and applied population proportional allocation according to the 5 major areas of Seoul and type of residence. The subjects were limited to adults aged 19 and over. This study was approved by the Institutional Review Board of Seoul Medical Center (IRB no. 2018-05-006).

\section{Survey}

As of early July $2018,191,850$ out of 283,685 participants in the Seoul registered survey company were randomly extracted and sent e-mail requesting participation through an email program. 25,285 out of 191,850 subjects confirmed via e-mail, and 5,934 of them accessed the online survey. However, 656 subjects were excluded through the screening, 3,424 subjects were excluded due to over-representation of proportional allocation, and 156 subjects were excluded due to abandonment, thus only 1,698 subjects completed the survey. Of these, 198 subjects who repeatedly responded to a certain interval or had a short response time were excluded. Ultimately, 1,500 subjects were included in the analysis. Since the subjects are panel members managed by the survey company, they paid for itself in the form of mileage to the subjects.

\section{Generall Characteristics}

The general characteristics of survey included the following: gender (male, female), age (20-29 years, 30-39 years, 40-49 years, $\geq 50$ years), area (downtown, northeast, northwest, southeast, southwest), residence period (1-3 years, 3-5 years, $\geq 5$ years), residence type (detached house, apartment, multiplex house, commercial house), marital status (single, married), education ( $\leq$ high school, university, $\geq$ graduate school), occupation (production, professional, office work, sales/service, self-employed, student, housewife, unemployed), monthly household income ( $<2$ million won, 2-5 million won, 5-7 million won, $\geq 7$ million won), number of residents ( 1 person, 2 people, 3 people, 4 people, $\geq 5$ people), household type (1 generation, 2 generations, $\geq 3$ generations, non-kin, 1 person), minor child (no, yes), residence scale $\left(\leq 60 \mathrm{~m}^{2}, 60-85 \mathrm{~m}^{2}\right.$, $85-135 \mathrm{~m}^{2},>135 \mathrm{~m}^{2}$ ).

\section{The use of HPs at home}

HPs were selected as 23 kinds of products with associated risks that are management targets of the ME [16]. Products with associated risks are classified as follows in accordance with standard guided by ME: 5 kinds of detergents (cleaner, synthetic detergent, bleaching agent, softening agent, windshield washer fluid), 6 kinds of coatings/adhesives (coating, rust inhibitor, anti-fogging agent, adhesive, ironing auxiliaries, niche filler), 2 kinds of aromatic products (air freshener, deodorant), 3 kinds of dyes (object decolorant and dye, tattoo dye, printing ink and toner) 4 kinds of biocidal products (disinfectant, insect repellent, preservative, algicide), 3 kinds of others (candle, desiccant, antifreeze). In the survey, the use (used, not used) and usage frequency (1-11 times a year, 1-3 times a month, 1-6 times a week, 1-4 times a day) of each product for the last 1 year were examined. If the subject used the product more than 4 times a day, they were asked to fill in 
the number directly. Usage frequency of each product was quantitatively converted to a per month figure.

\section{Status of AD}

The status of $\mathrm{AD}$ was assessed using the International Study of Asthma and Allergies in Childhood questionnaires. The survey included the following information related to $\mathrm{AD}$, indoor environmental factors, and family history: lifetime diagnosis of $\mathrm{AD}$ (no, yes), symptoms of AD within the last 12 months (no, yes), treatment of $\mathrm{AD}$ within the last 12 months (no, yes), current smoking (no, past experience, yes), indoor secondhand smoking (no, 1-2 times a week, 3-4 times a week, 5-6 times a week, everyday), remodeling in the last 12 months (no, yes), use of new furniture in the last 12 months (no, yes), mold stain in current residence (no, yes), pets in current residence (no, yes), parental lifetime diagnosis of allergic diseases (no, yes).

\section{Statistics}

The number of used HPs was represented by the total number of HPs who answered that they use each product, and usage frequency was expressed as arithmetic mean and standard deviation (SD) using average usage frequency for each product. These were divided into quartiles in the analysis of $\mathrm{AD}$. General characteristics, indoor environmental factors, family history, and number of used HPs and usage frequency were subjected to a Chi-square test. To assess the relationship between the use of HPs and AD, logistic regression analysis was conducted after correcting for general characteristics, indoor environmental factors, and family history of this study. These results were shown by odds ratio (OR) and had a $95 \%$ confidence interval (CI). All of the statistics were performed using Statistical Analysis Software (SPSS version 18.0, SPSS Inc.).

\section{RESULTS}

\section{Usage Status of HPs}

Average number of used HPs and average usage frequency of HPs of 1,500 households in Seoul were 13.44 out of 23 and 3.52 times a month, respectively (Table 1). Frequency distribution of number of used HPs are presented in Table S1. When the use of HPs was divided into quartiles, the number of used HPs was $\leq 10$ in the 1st quartile, 11-13 in the 2nd quartile, 1416 in the 3rd quartile, and $\geq 17$ in the 4 th quartile, and the usage frequency was $\leq 1.32$ times a month in the 1st quartile, 1.32-2.52 times a month in the 2nd quartile, 2.52-4.24 times a month in the 3rd quartile, and $>4.24$ times a month in the 4 th quartile.

\section{Related Factors of AD}

Table 2 showed general characteristics, indoor environmental factors, family history, and the use distribution of HPs of the subjects according to AD. The percentage of adults with the experience of $\mathrm{AD}$ diagnosis in their lifetime was significantly higher than those without, as follows: $28.8 \%$ for those aged $20-29$ years $(\mathrm{p}<0.05), 48.3 \%$ for those who were single $(\mathrm{p}<0.05), 18.8 \%$ for those who had a history of smoking ( $\mathrm{p}<0.05), 22.1 \%$ for those with daily indoor secondhand smoke exposure ( $\mathrm{p}<0.01), 32.6 \%$ for those who had remodeled in the last 12 months $(\mathrm{p}<0.001), 61.7 \%$ for those with mold stain in the current residence $(\mathrm{p}<0.001), 23.3 \%$ for those with pets in the current residence ( $\mathrm{p}<0.001), 45.7 \%$ for those with a parental lifetime diagnosis of allergic diseases $(\mathrm{p}<0.001), 29.8 \%$ for number of used HPs in the 4 th quartile $(p<0.001), 32.6 \%$ for usage frequency of HPs in the 4 th quartile $(\mathrm{p}<0.001)$. Adults with the experience of symptoms of $\mathrm{AD}$ within the last 12 months were significantly higher than those without the experience of $\mathrm{AD}$ in the following groups: $52.9 \%$ for females $(\mathrm{p}<0.05), 14.7 \%$ for $\leq$ high school graduation $(\mathrm{p}<0.05), 20.9 \%$ for those with a residence size $\leq 60 \mathrm{~m}^{2}(\mathrm{p}<0.05), 24.1 \%$ for those with daily indoor secondhand smoke exposure $(\mathrm{p}<0.001)$, $31.8 \%$ for those who had remodeled in the last 12 months $(\mathrm{p}<0.001), 38.8 \%$ for those with new furniture in the last 12 months ( $\mathrm{p}<0.001), 59.9 \%$ for those with a mold stain in their current residence $(\mathrm{p}<0.001), 20.2 \%$ for those with pets in their current residence $(\mathrm{p}<0.05), 38.8 \%$ for those with a parental lifetime diagnosis of allergic diseases $(\mathrm{p}<0.001), 26.6 \%$ for number of used HPs in the 4 th quartile $(\mathrm{p}<0.001), 29.6 \%$ for usage frequency of HPs in the 4 th quartile $(\mathrm{p}<0.001)$. Adults with the experience of treatment of $\mathrm{AD}$ within the last 12 months were significantly higher than those without in the following groups: $21.2 \%$ for those with graduate school educa-

Table 1. Usage distribution of household products (HPS)

\begin{tabular}{|c|c|c|c|c|c|c|c|}
\hline \multirow{2}{*}{ Variables } & \multirow{2}{*}{$\mathrm{N}$} & \multirow{2}{*}{ Mean } & \multirow{2}{*}{$\mathrm{SD}^{*}$} & \multicolumn{4}{|c|}{ Percentiles } \\
\hline & & & & 25th & 50th & 75th & 95th \\
\hline Number of used HPs (number/year) & 1,500 & 13.44 & 4.82 & 10.00 & 13.00 & 16.00 & 23.00 \\
\hline Usage frequency of HPs (times/month) & 1,500 & 3.52 & 4.23 & 1.32 & 2.52 & 4.24 & 9.75 \\
\hline
\end{tabular}

${ }^{*} \mathrm{SD}=$ Standard deviation. 
Table 2. Summary on characteristics of subjects for atopic dermatitis (AD)

\begin{tabular}{|c|c|c|c|c|c|c|c|c|c|c|c|c|c|c|c|c|}
\hline & \multirow{3}{*}{ Total } & \multicolumn{5}{|c|}{ Diagnosis, lifetime } & \multicolumn{5}{|c|}{ Symptoms, $<12$ months } & \multicolumn{5}{|c|}{ Treatment, $<12$ months } \\
\hline & & \multicolumn{2}{|c|}{ No } & \multicolumn{2}{|c|}{ Yes } & \multirow{2}{*}{$p$-value } & \multicolumn{2}{|c|}{ No } & \multicolumn{2}{|c|}{ Yes } & \multirow{2}{*}{$p$-value } & \multicolumn{2}{|c|}{ No } & \multicolumn{2}{|c|}{ Yes } & n \\
\hline & & $\mathrm{N}$ & $\%$ & $\mathrm{~N}$ & $\%$ & & $\mathrm{~N}$ & $\%$ & $\mathrm{~N}$ & $\%$ & & $\mathrm{~N}$ & $\%$ & N & $\%$ & $p$-value \\
\hline Total & 1,500 & 1,080 & 72.0 & 420 & 28.0 & & 774 & 51.6 & 726 & 48.4 & & 1,269 & 84.6 & 231 & 15.4 & \\
\hline Gender & & & & & & & & & & & & & & & & \\
\hline Male & 750 & 538 & 49.8 & 212 & 50.5 & 0.82 & 408 & 52.7 & 342 & 47.1 & $<0.05$ & 637 & 50.2 & 113 & 48.9 & 0.72 \\
\hline Female & 750 & 542 & 50.2 & 208 & 49.5 & & 366 & 47.3 & 384 & 52.9 & & 632 & 49.8 & 118 & 51.1 & \\
\hline Age (years) & & & & & & & & & & & & & & & & \\
\hline 20-29 & 350 & 229 & 21.2 & 121 & 28.8 & $<0.05$ & 194 & 25.1 & 156 & 21.5 & 0.27 & 286 & 22.5 & 64 & 27.7 & 0.33 \\
\hline $30-39$ & 400 & 296 & 27.4 & 104 & 24.8 & & 205 & 26.5 & 195 & 26.9 & & 343 & 27.1 & 57 & 24.7 & \\
\hline $40-49$ & 400 & 289 & 26.8 & 111 & 26.4 & & 193 & 24.9 & 207 & 28.5 & & 338 & 26.6 & 62 & 26.8 & \\
\hline$\geq 50$ & 350 & 266 & 24.6 & 84 & 20.0 & & 182 & 23.5 & 168 & 23.1 & & 302 & 23.8 & 48 & 20.8 & \\
\hline Area & & & & & & & & & & & & & & & & \\
\hline Downtown & 81 & 54 & 5.0 & 27 & 6.4 & 0.40 & 41 & 5.3 & 40 & 5.5 & 0.77 & 65 & 5.1 & 16 & 6.9 & 0.81 \\
\hline Northeast & 466 & 333 & 30.9 & 133 & 31.7 & & 231 & 29.8 & 235 & 32.4 & & 393 & 31.0 & 73 & 31.6 & \\
\hline Northwest & 187 & 144 & 13.3 & 43 & 10.2 & & 102 & 13.2 & 85 & 11.7 & & 161 & 12.7 & 26 & 11.3 & \\
\hline Southeast & 326 & 238 & 22.0 & 88 & 21.0 & & 167 & 21.6 & 159 & 21.9 & & 277 & 21.8 & 49 & 21.2 & \\
\hline Southwest & 440 & 311 & 28.8 & 129 & 30.7 & & 233 & 30.1 & 207 & 28.5 & & 373 & 29.4 & 67 & 29.0 & \\
\hline Residence period (ye & & & & & & & & & & & & & & & & \\
\hline $1-3$ & 59 & 37 & 3.4 & 22 & 5.2 & 0.16 & 34 & 4.4 & 25 & 3.4 & 0.53 & 48 & 3.8 & 11 & 4.8 & 0.59 \\
\hline $3-5$ & 53 & 35 & 3.2 & 18 & 4.3 & & 25 & 3.2 & 28 & 3.9 & & 43 & 3.4 & 10 & 4.3 & \\
\hline$\geq 5$ & 1,388 & 1,008 & 93.4 & 380 & 90.5 & & 715 & 92.4 & 673 & 92.7 & & 1,178 & 92.8 & 210 & 90.9 & \\
\hline Residence type & & & & & & & & & & & & & & & & \\
\hline Detached house & 180 & 125 & 11.6 & 55 & 13.1 & 0.35 & 86 & 11.1 & 94 & 12.9 & 0.43 & 145 & 11.4 & 35 & 15.2 & 0.16 \\
\hline Apartment & 887 & 654 & 60.6 & 233 & 55.5 & & 473 & 61.1 & 414 & 57.1 & & 748 & 58.9 & 139 & 60.2 & \\
\hline Multiplex house & 415 & 289 & 26.8 & 126 & 30.0 & & 206 & 26.6 & 209 & 28.8 & & 362 & 28.6 & 53 & 22.9 & \\
\hline Commercial house & 18 & 12 & 1.0 & 6 & 1.4 & & 9 & 1.2 & 9 & 1.2 & & 14 & 1.1 & 4 & 1.7 & \\
\hline Marital status & & & & & & & & & & & & & & & & \\
\hline Single & 652 & 449 & 41.6 & 203 & 48.3 & $<0.05$ & 342 & 44.2 & 310 & 42.7 & 0.56 & 546 & 43.0 & 106 & 45.9 & 0.42 \\
\hline Married & 848 & 631 & 58.4 & 217 & 51.7 & & 432 & 55.8 & 416 & 57.3 & & 723 & 57.0 & 125 & 54.1 & \\
\hline Education & & & & & & & & & & & & & & & & \\
\hline$\leq$ High school & 182 & 134 & 12.4 & 48 & 11.4 & 0.24 & 75 & 9.7 & 107 & 14.7 & $<0.05$ & 157 & 12.4 & 25 & 10.8 & $<0.05$ \\
\hline University & 1,081 & 786 & 72.8 & 295 & 70.3 & & 576 & 74.4 & 505 & 69.6 & & 924 & 72.8 & 157 & 68.0 & \\
\hline$\geq$ Graduate school & 237 & 160 & 14.8 & 77 & 18.3 & & 123 & 15.9 & 114 & 15.7 & & 188 & 14.8 & 49 & 21.2 & \\
\hline Occupation & & & & & & & & & & & & & & & & \\
\hline Production & 21 & 14 & 1.3 & 7 & 1.6 & 0.76 & 10 & 1.2 & 11 & 1.5 & 0.29 & 16 & 1.2 & 5 & 2.1 & 0.16 \\
\hline Professional & 211 & 148 & 13.7 & 63 & 15.0 & & 109 & 14.1 & 102 & 14.0 & & 179 & 14.1 & 32 & 13.9 & \\
\hline Office work & 701 & 513 & 47.5 & 188 & 44.8 & & 360 & 46.5 & 341 & 47.0 & & 601 & 47.4 & 100 & 43.3 & \\
\hline Sales/Service & 77 & 57 & 5.3 & 20 & 4.8 & & 42 & 5.4 & 35 & 4.8 & & 68 & 5.4 & 9 & 3.9 & \\
\hline Self-employed & 93 & 66 & 6.1 & 27 & 6.4 & & 45 & 5.8 & 48 & 6.6 & & 73 & 5.8 & 20 & 8.7 & \\
\hline Student & 145 & 96 & 8.9 & 49 & 11.7 & & 88 & 11.4 & 57 & 7.9 & & 119 & 9.4 & 26 & 11.3 & \\
\hline Housewife & 185 & 136 & 12.6 & 49 & 11.7 & & 84 & 10.9 & 101 & 13.9 & & 151 & 11.9 & 34 & 14.7 & \\
\hline Unemployed & 67 & 50 & 4.6 & 17 & 4.0 & & 36 & 4.7 & 31 & 4.3 & & 62 & 4.8 & 5 & 2.1 & \\
\hline $\begin{array}{l}\text { Household income } \\
\text { (million won/month) }\end{array}$ & & & & & & & & & & & & & & & & \\
\hline$<2$ & 104 & 76 & 7.0 & 28 & 6.6 & 0.84 & 53 & 6.8 & 51 & 7.0 & 0.64 & 93 & 7.3 & 11 & 4.7 & $<0.05$ \\
\hline $2-5$ & 743 & 529 & 49.0 & 214 & 51.0 & & 390 & 50.4 & 353 & 48.7 & & 638 & 50.3 & 105 & 45.5 & \\
\hline $5-7$ & 365 & 269 & 24.9 & 96 & 22.9 & & 192 & 24.8 & 173 & 23.8 & & 310 & 24.4 & 55 & 23.8 & \\
\hline$\geq 7$ & 288 & 206 & 19.1 & 82 & 19.5 & & 139 & 18.0 & 149 & 20.5 & & 228 & 18.0 & 60 & 26.0 & \\
\hline Number of residents & & & & & & & & & & & & & & & & \\
\hline 1 & 153 & 109 & 10.1 & 44 & 10.5 & 0.35 & 73 & 9.4 & 80 & 11.0 & 0.52 & 133 & 10.5 & 20 & 8.7 & 0.13 \\
\hline 2 & 236 & 176 & 16.3 & 60 & 14.3 & & 127 & 16.4 & 109 & 15.0 & & 208 & 16.4 & 28 & 12.1 & \\
\hline 3 & 447 & 331 & 30.6 & 116 & 27.6 & & 242 & 31.3 & 205 & 28.2 & & 383 & 30.2 & 64 & 27.7 & \\
\hline 4 & 544 & 385 & 35.7 & 159 & 37.9 & & 271 & 35.0 & 273 & 37.7 & & 449 & 35.4 & 95 & 41.1 & \\
\hline$\geq 5$ & 120 & 79 & 7.3 & 41 & 9.7 & & 61 & 7.9 & 59 & 8.1 & & 96 & 7.5 & 24 & 10.4 & \\
\hline Household type & & & & & & & & & & & & & & & & \\
\hline 1 Generation & 262 & 191 & 17.7 & 71 & 16.9 & 0.92 & 146 & 18.9 & 116 & 16.0 & 0.40 & 228 & 18.0 & 34 & 14.7 & 0.59 \\
\hline 2 Generations & 971 & 702 & 65.0 & 269 & 64.0 & & 495 & 64.0 & 476 & 65.6 & & 812 & 64.0 & 159 & 68.8 & \\
\hline$\geq 3$ Generations & 97 & 66 & 6.1 & 31 & 7.4 & & 49 & 6.3 & 48 & 6.6 & & 81 & 6.4 & 16 & 6.9 & \\
\hline
\end{tabular}




\begin{tabular}{|c|c|c|c|c|c|c|c|c|c|c|c|c|c|c|c|c|}
\hline & \multirow{3}{*}{ Total } & \multicolumn{5}{|c|}{ Diagnosis, lifetime } & \multicolumn{5}{|c|}{ Symptoms, $<12$ months } & \multicolumn{5}{|c|}{ Treatment, $<12$ months } \\
\hline & & \multicolumn{2}{|c|}{ No } & \multicolumn{2}{|c|}{ Yes } & \multirow{2}{*}{$p$-value } & \multicolumn{2}{|c|}{ No } & \multicolumn{2}{|c|}{ Yes } & \multirow{2}{*}{$p$-value } & \multicolumn{2}{|c|}{ No } & \multicolumn{2}{|c|}{ Yes } & \multirow{2}{*}{$p$-value } \\
\hline & & $\mathrm{N}$ & $\%$ & $\mathrm{~N}$ & $\%$ & & $\mathrm{~N}$ & $\%$ & $\mathrm{~N}$ & $\%$ & & N & $\%$ & N & $\%$ & \\
\hline Non-kin & 17 & 12 & 1.1 & 5 & 1.2 & & 11 & 1.4 & 6 & 0.8 & & 15 & 1.1 & 2 & 0.9 & \\
\hline 1 Person & 153 & 109 & 10.1 & 44 & 10.5 & & 73 & 9.4 & 80 & 11.0 & & 133 & 10.5 & 20 & 8.7 & \\
\hline \multicolumn{17}{|l|}{ Minor child } \\
\hline No & 1,033 & 746 & 69.1 & 287 & 68.3 & 0.78 & 548 & 70.8 & 485 & 66.8 & 0.10 & 883 & 69.6 & 150 & 64.9 & 0.16 \\
\hline Yes & 467 & 334 & 30.9 & 133 & 31.7 & & 226 & 29.2 & 241 & 33.2 & & 386 & 30.4 & 81 & 35.1 & \\
\hline \multicolumn{17}{|c|}{ Residence scale $\left(\mathrm{m}^{2}\right)$} \\
\hline$\leq 60$ & 288 & 211 & 19.5 & 77 & 18.2 & 0.56 & 136 & 17.6 & 152 & 20.9 & $<0.05$ & 257 & 20.3 & 31 & 13.4 & $<0.01$ \\
\hline $60-85$ & 425 & 308 & 28.5 & 117 & 27.9 & & 240 & 31.0 & 185 & 25.5 & & 368 & 29.0 & 57 & 24.7 & \\
\hline $85-135$ & 648 & 468 & 43.4 & 180 & 42.9 & & 337 & 43.5 & 311 & 42.9 & & 534 & 42.1 & 114 & 49.4 & \\
\hline$>135$ & 139 & 93 & 8.6 & 46 & 11.0 & & 61 & 7.9 & 78 & 10.7 & & 110 & 8.6 & 29 & 12.5 & \\
\hline \multicolumn{17}{|l|}{ Smoking } \\
\hline No & 947 & 692 & 64.1 & 255 & 60.7 & $<0.05$ & 502 & 64.9 & 445 & 61.3 & 0.13 & 809 & 63.8 & 138 & 59.7 & 0.28 \\
\hline Past experience & 223 & 144 & 13.3 & 79 & 18.8 & & 118 & 15.2 & 105 & 14.5 & & 181 & 14.2 & 42 & 18.2 & \\
\hline Yes & 330 & 244 & 22.6 & 86 & 20.5 & & 154 & 19.9 & 176 & 24.2 & & 279 & 22.0 & 51 & 22.1 & \\
\hline \multicolumn{17}{|c|}{$\begin{array}{l}\text { Secondhand smoke } \\
\text { exposure (times/week) }\end{array}$} \\
\hline No & 282 & 229 & 21.2 & 53 & 12.6 & $<0.01$ & 185 & 23.9 & 97 & 13.4 & $<0.001$ & 255 & 20.1 & 27 & 11.7 & $<0.05$ \\
\hline $1-2$ & 590 & 427 & 39.5 & 163 & 38.8 & & 308 & 39.8 & 282 & 38.8 & & 502 & 39.6 & 88 & 38.1 & \\
\hline $3-4$ & 231 & 153 & 14.2 & 78 & 18.6 & & 112 & 14.5 & 119 & 16.4 & & 188 & 14.8 & 43 & 18.6 & \\
\hline $5-6$ & 106 & 73 & 6.8 & 33 & 7.9 & & 53 & 6.8 & 53 & 7.3 & & 88 & 6.9 & 18 & 7.8 & \\
\hline Daily & 291 & 198 & 18.3 & 93 & 22.1 & & 116 & 15.0 & 175 & 24.1 & & 236 & 18.6 & 55 & 23.8 & \\
\hline Remodeling & & & & & & & & & & & & & & & & \\
\hline No & 1,112 & 829 & 76.8 & 283 & 67.4 & $<0.001$ & 617 & 79.7 & 495 & 68.2 & $<0.001$ & 968 & 76.3 & 144 & 62.3 & $<0.001$ \\
\hline Yes & 388 & 251 & 23.2 & 137 & 32.6 & & 157 & 20.3 & 231 & 31.8 & & 301 & 23.7 & 87 & 37.7 & \\
\hline New furniture & & & & & & & & & & & & & & & & \\
\hline No & 995 & 729 & 67.5 & 266 & 63.3 & 0.13 & 551 & 71.2 & 444 & 61.2 & $<0.001$ & 866 & 68.2 & 129 & 55.8 & $<0.001$ \\
\hline Yes & 505 & 351 & 32.5 & 154 & 36.7 & & 223 & 28.8 & 282 & 38.8 & & 403 & 31.8 & 102 & 44.2 & \\
\hline Mold stain & & & & & & & & & & & & & & & & \\
\hline No & 703 & 542 & 50.2 & 161 & 38.3 & $<0.001$ & 412 & 53.2 & 291 & 40.1 & $<0.001$ & 620 & 48.9 & 83 & 35.9 & $<0.001$ \\
\hline Yes & 797 & 538 & 49.8 & 259 & 61.7 & & 362 & 46.8 & 435 & 59.9 & & 649 & 51.1 & 148 & 64.1 & \\
\hline Pets & & & & & & & & & & & & & & & & \\
\hline No & 1,235 & 913 & 84.5 & 322 & 76.7 & $<0.001$ & 656 & 84.8 & 579 & 79.8 & $<0.05$ & 1,068 & 84.2 & 167 & 72.3 & $<0.001$ \\
\hline Yes & 265 & 167 & 15.5 & 98 & 23.3 & & 118 & 15.2 & 147 & 20.2 & & 201 & 15.8 & 64 & 27.7 & \\
\hline Family history & & & & & & & & & & & & & & & & \\
\hline No & 1,066 & 838 & 77.6 & 228 & 54.3 & $<0.001$ & 622 & 80.4 & 444 & 61.2 & $<0.001$ & 964 & 76.0 & 102 & 44.2 & $<0.001$ \\
\hline Yes & 434 & 242 & 22.4 & 192 & 45.7 & & 152 & 19.6 & 282 & 38.8 & & 305 & 24.0 & 129 & 55.8 & \\
\hline Number of used HP & & & & & & & & & & & & & & & & \\
\hline $1^{\text {st }}$ Quartile & 426 & 336 & 31.2 & 90 & 21.4 & $<0.001$ & 275 & 35.6 & 151 & 20.8 & $<0.001$ & 390 & 30.7 & 36 & 15.6 & $<0.001$ \\
\hline $2^{\text {nd }}$ Quartile & 405 & 294 & 27.2 & 111 & 26.4 & & 212 & 27.4 & 193 & 26.6 & & 349 & 27.5 & 56 & 24.2 & \\
\hline $3^{\text {rd }}$ Quartile & 317 & 223 & 20.6 & 94 & 22.4 & & 128 & 16.5 & 189 & 26.0 & & 264 & 20.8 & 53 & 22.9 & \\
\hline $4^{\text {th }}$ Quartile & 352 & 227 & 21.0 & 125 & 29.8 & & 159 & 20.5 & 193 & 26.6 & & 266 & 21.0 & 86 & 37.3 & \\
\hline Usage frequency 0 & & & & & & & & & & & & & & & & \\
\hline $1^{\text {st }}$ Quartile & 375 & 302 & 28.0 & 73 & 17.4 & $<0.001$ & 251 & 32.4 & 124 & 17.1 & $<0.001$ & 343 & 27.0 & 32 & 13.8 & $<0.001$ \\
\hline $2^{\text {nd }}$ Quartile & 373 & 259 & 24.0 & 114 & 27.1 & & 186 & 24.0 & 187 & 25.8 & & 318 & 25.1 & 55 & 23.8 & \\
\hline $3^{\text {rd }}$ Quartile & 377 & 281 & 26.0 & 96 & 22.9 & & 177 & 22.9 & 200 & 27.5 & & 320 & 25.2 & 57 & 24.7 & \\
\hline $4^{\text {th }}$ Quartile & 375 & 238 & 22.0 & 137 & 32.6 & & 160 & 20.7 & 215 & 29.6 & & 288 & 22.7 & 87 & 37.7 & \\
\hline
\end{tabular}

tion or higher $(\mathrm{p}<0.05), 26.0 \%$ for those with a household income of 7 million won per month or more $(\mathrm{p}<0.05), 12.5 \%$ for those with a residence less than $135 \mathrm{~m}^{2}(\mathrm{p}<0.01), 23.8 \%$ for those with everyday indoor secondhand smoke exposure ( $\mathrm{p}<0.05), 37.7 \%$ for those who had remodeled in the last 12 months $(\mathrm{p}<0.001), 44.2 \%$ for those that had used new furniture in the last 12 months ( $\mathrm{p}<0.001), 64.1 \%$ for those with a mold stain in their current residence $(\mathrm{p}<0.001), 27.7 \%$ for those with pets in their current residence $(\mathrm{p}<0.05), 55.8 \%$ for those with a parental lifetime diagnosis of allergic diseases $(\mathrm{p}<0.001)$, $37.3 \%$ for number of used HPs in the 4 th quartile $(\mathrm{p}<0.001)$, $37.7 \%$ for usage frequency of HPs in the 4 th quartile $(\mathrm{p}<0.001)$. 
Table 3. Logistic regression analysis of number of household products (HPs) used and atopic dermatitis (AD)

\begin{tabular}{|c|c|c|c|c|c|c|}
\hline & \multicolumn{2}{|c|}{ Diagnosis, lifetime } & \multicolumn{2}{|c|}{ Symptoms, $<12$ months } & \multicolumn{2}{|c|}{ Treatment, $<12$ months } \\
\hline & $\mathrm{OR}^{\star}\left(95 \% \mathrm{Cl}^{\dagger}\right)$ & $\mathrm{p}$-value & $\mathrm{OR}^{\star}\left(95 \% \mathrm{Cl}^{\dagger}\right)$ & $p$-value & $\mathrm{OR}^{\star}\left(95 \% \mathrm{Cl}^{\dagger}\right)$ & $p$-value \\
\hline $1^{\text {st }}$ Quartile & 1 & & 1 & & 1 & \\
\hline $2^{\text {nd }}$ Quartile & $1.42(1.01-2.01)$ & $<0.05$ & $1.65(1.22-2.23)$ & $<0.01$ & $1.58(0.98-2.53)$ & 0.06 \\
\hline $3^{\text {rd }}$ Quartile & $1.46(1.01-2.10)$ & $<0.05$ & $1.93(1.39-2.69)$ & $<0.001$ & $1.76(1.08-2.86)$ & $<0.05$ \\
\hline $4^{\text {th }}$ Quartile & $1.77(1.23-2.54)$ & $<0.01$ & $2.66(1.92-3.70)$ & $<0.001$ & $2.37(1.48-3.80)$ & $<0.001$ \\
\hline$p$ for trend & \multicolumn{2}{|c|}{$<0.01$} & \multicolumn{2}{|c|}{$<0.001$} & \multicolumn{2}{|c|}{$<0.001$} \\
\hline
\end{tabular}

${ }^{*}$ Odds ratio, ${ }^{\dagger}$ Confidence interval.

Adjusted variables for gender, age, area, residence period, residence type, marital status, education, occupation, household income, number of residents, household type, minor child, residence scale, smoking, secondhand smoke exposure, remodeling, new furniture, mold stain, pets and family history.

Table 4. Logistic regression analysis of usage frequency of household products (HPS) and atopic dermatitis (AD)

\begin{tabular}{|c|c|c|c|c|c|c|}
\hline & \multicolumn{2}{|c|}{ Diagnosis, lifetime } & \multicolumn{2}{|c|}{ Symptoms, $<12$ months } & \multicolumn{2}{|c|}{ Treatment, $<12$ months } \\
\hline & $\mathrm{OR}^{\star}\left(95 \% \mathrm{Cl}^{\dagger}\right)$ & $p$-value & $\mathrm{OR}^{\star}\left(95 \% \mathrm{Cl}^{\dagger}\right)$ & $p$-value & $\mathrm{OR}^{*}\left(95 \% \mathrm{Cl}^{\dagger}\right)$ & $p$-value \\
\hline $1^{\text {st }}$ Quartile & 1 & & 1 & & 1 & \\
\hline $2^{\text {nd }}$ Quartile & $1.19(0.82-1.72)$ & 0.37 & $1.84(1.34-2.53)$ & $<0.001$ & $1.40(0.85-2.30)$ & 0.19 \\
\hline $3^{\text {rd }}$ Quartile & $1.64(1.15-2.35)$ & $<0.01$ & $1.87(1.36-2.58)$ & $<0.001$ & $1.62(0.99-2.64)$ & 0.06 \\
\hline $4^{\text {th }}$ Quartile & $1.88(1.31-2.70)$ & $<0.01$ & $2.14(1.54-2.96)$ & $<0.001$ & $2.23(1.39-3.60)$ & $<0.01$ \\
\hline$p$ for trend & \multicolumn{2}{|c|}{$<0.01$} & \multicolumn{2}{|c|}{$<0.001$} & \multicolumn{2}{|c|}{$<0.01$} \\
\hline
\end{tabular}

${ }^{*}$ Odds ratio, ${ }^{\dagger}$ Confidence interval.

Adjusted variables for gender, age, area, residence period, residence type, marital status, education, occupation, household income, number of residents, household type, minor child, residence scale, smoking, secondhand smoke exposure, remodeling, new furniture, mold stain, pets and family history.

\section{Relationship Between Number of Used HPs and AD}

In order to evaluate the relationship between the number of used HPs and AD, a logistic regression analysis was performed by adjusting the confounding variables. The results are shown in Table 3. The risk of lifetime diagnosis of $\mathrm{AD}$ compared to the 1st quartile was 1.42 times higher (95\% CI; 1.01-2.01) in the 2nd quartile, 1.46 times higher (95\% CI; 1.01-2.10) in the 3rd quartile, and 1.77 times higher (95\% CI; 1.23-2.54) in the 4th quartile. The risk of symptoms of $\mathrm{AD}$ within the last 12 months was 1.65 times higher (95\% CI; 1.22-2.23) in the 2nd quartile, 1.93 times higher (95\% CI; 1.39-2.69) in the 3rd quartile, and 2.66 times higher (95\% CI; 1.92-3.70) in the 4th quartile. The risk of treatment of $\mathrm{AD}$ within the last 12 months was 1.76 times (95\% CI; 1.08-2.86) higher in the 3rd quartile, and 2.37 times higher (95\% CI; 1.48-3.80) in the 4 th quartile. As the number of used HPs increased, the risks of lifetime diagnosis $(\mathrm{p}<0.01)$, symptoms within the last 12 months $(\mathrm{p}<0.001)$, and treatment of $\mathrm{AD}$ within the last 12 months $(\mathrm{p}<0.001)$ were increased significantly.

\section{Relationship Between Usage Frequency of HPs and AD}

As shown in Table 4, a logistic regression analysis was performed after adjusting for confounding variables to evaluate the relationship between the usage frequency of HPs and AD. Compared to the 1st quartile, the risk of lifetime diagnosis of $\mathrm{AD}$ was 1.64 times higher (95\% CI; 1.15-2.35) in the 3rd quartile, and 1.88 times higher (95\% CI; 1.31-2.70) in the 4 th quar- tile. The risk of symptoms of $\mathrm{AD}$ within the last 12 months was 1.84 times higher (95\% CI; 1.34-2.53) in the 2nd quartile, 1.87 times (95\% CI; 1.36-2.58) higher in the 3rd quartile, and 2.14 times higher (95\% CI; 1.54-2.96) in the 4th quartile. The risk of treatment of $\mathrm{AD}$ within the last 12 months was 2.23 times higher (95\% CI; 1.39-3.60) in the 4th quartile. As usage frequency of HPs increased, the risk of lifetime diagnosis $(\mathrm{p}<0.01)$, symptoms within the last 12 months $(\mathrm{p}<0.001)$, and treatment within the last 12 months $(\mathrm{p}<0.01)$ of $\mathrm{AD}$ was increased significantly. These results had a similar trend to the number of used HPs. Relationships between usage of 23 individual $\mathrm{HP}$ and $\mathrm{AD}$ are presented in Table $\mathrm{S} 2$.

\section{DISCUSSION}

This study of average number of used HPs and average usage frequency at home in Seoul for the last 1 year showed that consumers are still highly dependent on HPs. According to risk information trend analysis of household chemical HPs of KCA, accidents such as eye damage, internal dangerous substances, poisoning, burns, and pain at home were the most frequent type when risk was broken down by location [3]. This is thought to be related to the high use rates of HPs at home.

The experience rates of lifetime diagnosis of $\mathrm{AD}$, symptoms of $\mathrm{AD}$ within the last 12 months, and treatment of $\mathrm{AD}$ within the last 12 months in this study were higher than in a previous study [10]. This is because not only physical factors but also 
psychological factors such as stress and depression are reported to be involved in the onset of $\mathrm{AD}$ [17]. It is thought that those factors affect the continuous increase of experience rates of $\mathrm{AD}$.

In this study, $\mathrm{AD}$ was found to be associated with gender, age, marital status, education, household income, residence scale, smoking, indoor secondhand smoking, remodeling in the last 12 months, the use of new furniture in the last 12 months, mold stain, and parental lifetime diagnosis of allergic diseases. Previous studies on adults have reported the association of $\mathrm{AD}$ with gender, age, marital status, education, household income, number of residents, and residential area $[17,18]$, and the direction of relevance was consistent with this study. In particular, $\mathrm{AD}$ was significantly associated with parental family history [19], showing similar trend in this study. In addition, $\mathrm{AD}$ is also reported to be influenced by obesity, smoking, drinking, pets, stress, and dietary habits [20-23].

The findings of this study, which analyzed the relationship between the use of HPs and AD, shows that the risk of lifetime diagnosis of $\mathrm{AD}$, symptoms of $\mathrm{AD}$ within the last 12 months, and treatment of $\mathrm{AD}$ within the last 12 months was significantly increased as the number of used HPs and their usage frequency increased. It has been reported that surfactant or antimicrobial components contained in HPs cause allergy from skin contact and increase the risk of developing $\mathrm{AD}$ when it exposes continuously $[24,25,26]$, and this study supports this finding. In immunological mechanisms, harmful chemicals contained in HPs are exposed to antigens and the balance between T-helper type 1 (Th1) and T-helper type 2 (Th2) cytokine is broken, resulting in overproduced $\mathrm{Th} 2$ cytokines such as interleukin (IL)-4, 5, 9, 13 stimulating B cells and increasing immunoglobulin E (IgE) [27]. Increased IgE binds to receptors on the surface of mast cells and secretes chemicals such as histamine, stimulating blood vessels and skin which leads to $\mathrm{AD}$ [28]. In a previous domestic study, the risks of skin eczema were significantly increased according to usage frequency of antibacterial housewares, as follows: by 1.13 times (95\% CI; 1.04-1.24) in the 2nd quartile, by 1.15 times (95\% CI; 1.05-1.26) in the 3rd quartile, and by 1.13 times (95\% CI; 1.03-1.24) in the 4th quartile [29]. In an overseas study conducted through an online survey, $66.8 \%$ of the population was exposed to HPs at least 1 time a week due to the use of HPs, and $4.8 \%$ of them experienced skin symptoms [30]. A study of the effects of the use of disinfectants at home on allergic diseases found that the risk of $\mathrm{AD}$ was significantly higher when disinfectants were used, as follows: 2.6 times higher (95\% CI; 1.2-5.6) for lifetime symptoms of skin eczema, 7.0 times higher (95\% CI; 1.8-27.1) for symptoms of skin eczema within the last 12 months, and
2.5 times higher (95\% CI; 1.1-6.0) for lifetime symptoms of skin rash [31].

This study has a limitation in that it can be interpreted that there is the possibility of underestimation, because it includes subjects who responded that they did not use HPs in their usage frequency of HPs. In addition, the subjects responded to questionnaire relied on his or her memory to answer the online survey, so there is a possibility that the results are affected not only by reliability but also by recall bias. Nevertheless, the subjects of this study were applied population proportional allocation to represent households in Seoul, and this study shows that the risk of $\mathrm{AD}$ in adults was significantly increased as the number of used HPs and their usage frequency increased. Therefore, the findings of this study might be useful as basic data for managing allergic diseases and establishing preventive measures.

\section{ACKNOWLEDGEMENTS}

The authors thank Seoul Medical Center Research Institute, "A study on the use and exposure of hazardous substances contained in consumer products in the household, 2018 (18-A05)".

\section{CONFLICT OF INTEREST}

The authors have no conflicts of interest to declare in relation to the material presented in this paper.

\section{REFERENCES}

1. Lee S-M. Safety from danger of living chemical products. J Law Politics 2018; 18(3): 1-30. (Korean)

2. Lee J-S. New statutory framework for household chemical products in safety of household chemical products and biocidal products act. Hanyang J Law 2018; 35(3): 171-200. (Korean)

3. Korea Consumer Agency (KCA). Risk information trend analysis of household chemical products. 2017. (Korean)

4. National Institute of Environmental Research (NIER). Risk assessment for hazardous substances contained in consumer products. 2012. (Korean)

5. National Institute of Environmental Research (NIER). Studies on exposure assessment of nanomaterials in chemical products. 2017. (Korean)

6. Heo D-A, Huh E-H, Park JY, Moon KW, Lee K. An investigation of ingredients and hazardous substances in some consumer products: focusing on cleaners and disinfectants. J Environ Health Sci 2015; 41(5): 314-326. (Korean)

7. Steinemann AC, MacGregor IC, Gordon SM, Gallagher LG, Davis AL, Ribeiro DS, et al. Fragranced consumer products: chemicals emitted, ingredients unlisted. Environ Impact Assess Rev 2011; 31(3): 328-333.

8. Park DU, Lee S, Lim HK, Bae SY, Ryu SH, Ahn JJ. Review on safety and 
health information on humidifier disinfectant. J Environ Health Sci 2017; 43(5): 349-359. (Korean)

9. Wolkoff P, Schneider T, Kildeso J, Degerth R, Jaroszewski M, Schunk H. Risk in cleaning: chemical and physical exposure. Sci Total Environ 1998; 215(1-2): 135-156.

10. Kim MJ, Kang TW, Cho EA, Kim HS, Min JA, Park H, et al. Prevalence of atopic dermatitis among Korean adults visiting health service center of the catholic medical center in Seoul metropolitan area, Korea. J Korean Med Sci 2010; 25(12): 1828-1830.

11. Kim DH. Factors associated with behavioral problems in children with atopic dermatitis. J Korean Soc Matern Child Health 2010; 14: 206-214. (Korean)

12. Statistics Korea (KOSTAT). Korean Statistical Information Service (KOSIS). [cited 2019 March 14]. Available from: http://kosis.kr/statHtml/statHtml. do?orgId=117\&tblId=DT_11702_N114\&vw_cd=MT_ZTITLE\&list_ id=117_11702_B01\&seqNo=\&lang_mode=ko\&language=kor\&obj_var_ id=\&itm_id=\&conn_path=MT_ZTITLE.

13. Kim S-H, Park D-J, Byun H-J, Lee H-S, Oh I-B, Sim C-S, et al. House dust mites and associated environmental factors in homes of atopic children: a case-control study. J Environ Health Sci 2012; 38(3): 204212. (Korean)

14. Benn CS, Melbye M, Wohlfahrt J, Bjorksten B, Aaby P. Cohort study of sibling effect, infectious disease, and risk of atopic dermatitis during first 18 months of life. BMJ 2004; 328(7450): 1223-1226.

15. Katsarou A, Armenaka M. Atopic dermatitis in older patients: particular points. J Eur Acad Dermatol Venereol 2011; 25(1): 12-18.

16. Korea Consumer Agency (KCA). Survey on safety of children's protective packaging for household chemical products. 2017. (Korean)

17. Kim KH, Park AY, Kim JS. Factors associated with atopic dermatitis in Korean adults: The Korean National Health and Nutrition Survey 2008. Korean J Rehabil Nurs 2012; 15(2): 83-90. (Korean)

18. Kim BJ, Jung JA, Lee JS. Association between social economic status and atopic dermatitis in Korean adult: an analysis of the Fifth Korea National Health and Nutrition Examination Survey (2010-2012). Allergy Asthma Respir Dis 2015; 3(2): 128-133. (Korean)

19. Kim YM, Kim YC, Lee S, Back JH, Chun K. Association between parental history of allergic diseases and atopic dermatitis in school aged children. Allergy Asthma Respir Dis 2014; 2(5): 377-382. (Korean)
20. Lee YM, Hwang SW. Prevalence and risk factors for atopic dermatitis in pre-school and school aged children. J Korean Acad Child Health Nurs 2008; 14(3): 285-294. (Korean)

21. So ES, Yeo JY. Relationship between health status and life styles and atopic dermatitis in adolescents. J Korean Acad Child Health Nurs 2012; 18(3): 143-149. (Korean)

22. Park DH, Bae HS. Lifestyle habits and characteristics of atopic dermatitis occurred in children. Kor J Aesthet Cosmetol 2013; 11(1): 17-28. (Korean)

23. Park J-H. Factors influencing allergy related disease among Korean adolescents. J Kor Cont Assoc 2016; 16(3): 596-606. (Korean)

24. Jeong J, Jeong Y, Jeong S-W. Effects of bowl material and rinsing period on variation in the amount of residual surfactant resided in the bowls after dish-washing. J Kor Soc Environ Eng 2013; 35(12): 978981. (Korean)

25. Jung KH, Cho DW, Jung JH, Jeong NA, Joo KM, Seung KR, et al.Management directions for dangerous household goods. Duksung Bull Pharm Sci 2009; 20: 3-18. (Korean)

26. Ko JS. Antimicrobial coating agent. J Korean Oil Chem Soc 2013; 30(1): 96-115. (Korean)

27. Agnello D, Lankford CS, Bream J, Morinobu A, Gadina M, O'shea JJ, et al. Cytokines and transcription factors that regulate $\mathrm{T}$ helper cell differentiation: new players and new insights. J Clin Immunol 2003; 23(3): 147-161.

28. Lee B-W, Gim S-B, Song H-H, Ji J-G, Bak J-W, Kim D-H. Study on the immune modulatory activity of Seokjahaeki-tang using atopic dermatitis animal models. Korean J Orient Physiol Pathol 2012; 26(4): 446-454. (Korean)

29. Hong S, Kwon H-J, Choi W-J, Lim WR, Kim J, Kim K-S. Association between exposure to antimicrobial household products and allergic symptoms. Environ Health Toxicol 2014; 29: e2014017.

30. Steinemann A. Health and societal effects from exposure to fragranced consumer products. Prev Med Rep 2017; 5: 45-47.

31. Krauss-Etschmann S, Niedermaier S, Beyer J, Campoy C, Escolano V, Decsi T, et al. Current use of room disinfectants and allergic symptoms at the age of 4 years. J Allergy Clin Immunol 2009; 123(5): 11761178. 